\section{Longitudinal Study of \\ Periodontal Therapy}

by

SiguRd P. RAMF JORD**

JAMES W. KNOWLES**

ROBERT R. NISSLE**

RICHARD A. SHICK**

Frederick G. BurgetT**

\section{Pocket Depths and Attachment Levels*}

THIS PAPER IS A PROGRESS REPORT from an ongoing longitudinal study of periodontal therapy at The University of Michigan. A previous publication covered results from 1961 to $1966 .^{1}$ However, since the same patients are still participating in the study, we now have additional information on these and newer patients. The present report covers observations from the start of the study June 1, 1961 to January 1, 1972, including the data from our previous report.

Elimination of periodontal pockets is a common goal of periodontal therapy. With very few exceptions, it has been stated, or inferred, in the literature that periodontal pockets are self-perpetuating defects which sooner or later will lead to further loss of periodontal attachment. Evaluation of results from periodontal therapy has frequently been based on clinical reexamination of crevice depth at various time intervals following completion of the therapy, and with the implication that if the new crevice depth exceeded $3-4 \mathrm{~mm}$, the results of the therapy were unsatisfactory. With this philosophy in mind, various modalities of periodontal therapy have been developed for the purpose of elimination of periodontal crevices deeper than 2-3 mm. Some of these methods have undesirable side effects such as unsightly root exposure, thermal and mechanical sensitivity, root caries, and partial elimination of periodontal support. Only a demonstrable long-term benefit with regards to preservation of periodontal attachment would serve to justify such esthetic and functional sacrifices. One purpose of the present study has been to follow longitudinally, the interplay between degrees of return of pocket depth following various modalities of periodontal

*This research was supported in part by U.S.P.H.S., Grant \#DF-01430.

**The University of Michigan School of Dentistry, Ann Arbor, Michigan, 48104. therapy, and to relate these observations to variations in periodontal attachment levels in the same locations, in order to assess the significance of pocket elimination for maintenance of periodontal support.

The periodontium, by definition, constitutes the supporting system for the teeth. The two main purposes of periodontal therapy are to preserve supporting structures or gain lost support for the teeth, and to promote periodontal health.

Besides pocket depths and attachment levels, there are a number of other commonly used criteria for evaluation of results of periodontal therapy with main reference to periodontal health, such as clinical gingival status, oral hygiene status, and bone level. The interplay between these parameters and attachment levels are also being studied, but will be reported in subsequent papers.

The premise for the present study is that the single most important goal for periodontal therapy is to maintain or gain support for the teeth. Various techniques have been advocated to attain this goal. Most of these techniques also have pocket elimination as their goal. The efficacy of these techniques, or methods, toward long-term maintenance of attachment levels and pocket elimination has never been tested in a clinical trial. Neither has the principle of need for pocket elimination been tested with regards to its significance for maintenance of the periodontal support. Thus, the main purposes of the present investigation were:

1. To find which of two common principal approaches to periodontal therapy gives the best long-term results with regards to maintenance or gain of attachment levels.

2. To test the efficacy of these methods in long-term elimination of periodontal pockets.

3. To investigate if elimination of measurable periodontal pockets is essential for maintenance of periodontal attachment.

\section{Sample}

The limiting criteria for acceptance of patients for the project were that they had one or more periodontal pockets extending 4 millimeters or more apically to the cementum-enamel junction, and that they were interested and willing to come when called for examination and treatment. Teeth with pockets so close to the apex that they could not be treated periodontally without risk of pulp death, and endodontically or restoratively untreatable teeth were extracted. Otherwise, all degrees of severity of periodontal disease, from mild gingivitis to severe periodontitis with advanced bi- and tri-furcation 
involvements, were treated. Practically all of the patients had had some dental and a few, periodontal treatment; however, with poor results and/or stated poor or hopeless prognosis for their teeth. In some instances they were patients who could not afford proposed periodontal treatment in private practice.

A total of 124 patients have been treated since the project started June 1, 1961. At the present, (January 1,1972), 113 are actively participating. Twentyone patients have been lost from the project because of death (5), or moving away from the area (16). Six of these patients were lost after 1 year, 5 after 2 years, 3 after 3 years, 6 after 4 years, and 1 after 5 years. An analysis of this subset of lost patients indicated no statistical difference with the total sample or any trend toward systematic differences between this group and the total sample. The present report is based on 104 patients who have been treated during the last 10 years. Included are those patients who have been reexamined at least once, one year after completion of the periodontal treatment.

The range of age at the initiation of the treatment was 13 to 64 years, with a mean age of 39.7 years (S.D. 13.54). There were 50 females and 54 males. The average depth of the interproximal pockets at the distobuccal line angle of all teeth was $4.04 \mathrm{~mm}$. (S.D. $1.766)$, and at the mesio-buccal line angle $3.93 \mathrm{~mm}$. (S.D. 1.717). The average loss of attachment at the same location was respectively $3.53 \mathrm{~mm}$. (S.D. 2.140), and $3.14 \mathrm{~mm}$. (S.D. 2.011). A total of 2,604 teeth have been treated in these 104 patients, averaging approximately 25 teeth per patient.

\section{MeTHOD}

With some modifications in 1966 , we have followed the experimental design outlined in our previous publication. ${ }^{1}$

All patients initially have routine medical and dental history recorded, clinical examination, color photographs, long-cone technique dental roentgenograms, casts, scoring of: gingivitis, plaque and calculus, and measurements in millimeters of pocket depth and attachment levels at the mesial, distal, buccal, and lingual aspects of all teeth. ${ }^{1}$ Initial scorings and subsequent rescorings for the same patient have always been done by the same investigator. The three research associates (R.R.N., R.A.S., and F.G.B), working on the patients and doing the scoring, have been subjected to calibration tests several times during the project.

An analysis of variance was run on the data from the most recent calibration test performed on seven patients examined three times by each of the three examiners. This demonstrated no significance for either the between or the within examiner variation for any of the measurement variables. Also established was the small degree of error produced when patient measurements are carried out by the same examiner. To cite one example, the pooled estimate of patient standard deviation is 0.14 for mesial plus distal pocket depth and 0.17 for mesial plus distal attachment level which would then generate standard errors in the range of 0.02 for the number of patients in the present study. These errors are comparable to other published errors ${ }^{2,3}$ of similar measurements, and would apparently have an insignificant effect on the total results reported in the present paper.

The data from each scoring are kept on scoring sheets separate from the patient's records, so the examiner is not aware of the previous scores. The calibration tests and the data processing from the entire study are conducted by one investigator (J.W.K.) who has no contact with the patients or the therapeutic procedures.

After examination and scoring, all patients have initial treatment consisting of scaling, initial root planing, instruction in oral hygiene (no one standard method), and occlusal adjustment. They also receive emergency dental care including temporary fillings in advanced carious lesions and recommendations to have permanent restorations made.

\section{Experimental Treatment}

During the first two years of the study, approximately one-half of the patients were treated with curettage and the other half had surgical pocket elimination. An attempt was made to pair patients with similar periodontal status on an equal basis for the two experimental groups. However, it became evident that differences in oral hygiene and response from one patient to another would require a large number of randomized cases to equalize this variation. Also, unequal loss of patients in the two groups might change the character of the sample. Therefore the "split mouth" design was adopted using one side of the mouth for curettage and the other for surgical pocket elimination on a random basis so the hygienic and biologic difference would be minimized for the two experimental procedures.

Since 1963, the patients have been assigned to the experimental periodontal procedures on the basis of random number selection using the "split mouth" design whereby each patient is given different treatment in the right and left half of the dentition. In 1966, the experimental design was expanded to include a modified Widman (reverse bevel) flap procedure. The following three 
methods have thereafter been used for the experimental treatment: 1 . Curettage of the root surface and the soft tissue lining of the pocket under local anesthesia and with placement of a periodontal dressing following the treatment. (This method has not changed during the study.) 2. Surgical pocket elimination and contouring of the gingiva, either by gingivectomy or by an apically positioned reverse bevel full thickness flap. Osteoplasty or ostectomy is included as needed for pocket elimination and correction of architecture. Gingivectomy is preferred when the pockets are relatively shallow, are within the attached gingiva, and providing the pocket elimination will not involve bone surgery. The apically positioned flap has been used for all deep pockets, pockets of uneven depth and pockets requiring bone surgery for total eradication. (This method also has been unaltered during the entire study.) 3. Modified Widman flaps, which are scalloped reverse bevel mucoperiosteal flaps readapted to the neck of the teeth following removal of the epithelial lining and the soft connective tissues around the teeth, and down to the alveolar process but without any attempt to correct bony defect beyond what may be needed to achieve good flap adaptation to the teeth, and without any intentional repositioning of the flap in apical direction. (This method was introduced in 1966.)

The random assignment of the experimental treatment means that each half of the patient's dentition has an equal chance of being treated by any one of three procedures.

Following completion of the experimental treatment, the patient is placed on maintenance care which includes recall every three months for prophylaxis and further instruction in home care by a dental hygienist. One year after initiation of the experimental treatment, and annually thereafter, the patient is rescored using the same recording procedures as in the initial scoring, and new photographs and roentgenograms are obtained.

Only results from curettage and surgical pocket elimination are included in the present report.

\section{RESULTS}

The 104 patients included in the present report had 2604 teeth, of which 53 were lost during the study. Two of these teeth were lost strictly because of pulpal disease; three by accident; four for prosthetic considerations, and fourteen by one patient who after periodontal treatment desired a maxillary denture for cosmetic reasons. The remaining thirty lost teeth were extracted because of periodontal or combined periodontal and pulpal disease resulting in discomfort. A few teeth with very deep pockets became devital during the periodontal treatment or were discovered to be devital at the times of recall. Under these circumstances, it is difficult to assess whether the periodontal disease, combined with the treatment caused the pulpal involvement, or the pulpal involvement caused the apical extension of the pocket by retrograde periodontitis associated with infection of accessory canals in the furcation or apical regions. If a tooth was lost all previous data on this tooth was removed from the study to assure that the material analyzed at later years was compared with the same material that had been analyzed at the earlier years. Data from the lost teeth were analyzed for possible impact on the results of the present study. The majority of these teeth (32) were lost during the first and second year after initiation of treatment. The remaining 21 teeth were lost in a random pattern over the next 6 years. Twenty of the lost teeth had been treated by surgical pocket elimination, 19 by subgingival curettage and 9 by modified Widman flaps. The total loss of attachment (mesial + distal + buccal + lingual) was considerably greater for the lost teeth than for the total study population. The mean total loss of attachment was $0.52 \mathrm{~mm}$. with a standard deviation of 4.54 for one year of follow up; $2.03 \mathrm{~mm}$., S.D. 3.01 for two years; 2.25 mm., S.D. 3.36 for three years; $2.35 \mathrm{~mm}$., S.D. 3.27 for four years; $2.21 \mathrm{~mm}$., S.D. 2.21 for five years, and 6.85 mm., S.D. 3.97 for six years after initiation of the experimental treatment.

These values may be compared with the results from the entire experimental population listed later in this paper. Further characteristics of the lost teeth will be reported at a future date when larger material becomes available. Since the number of lost teeth never account for more than $1 \%$ of the number of teeth analyzed at any yearly interval, it was felt that the lost teeth would not influance the final interpretation of the results. The fact that teeth were lost from each of the two experimental groups in equal proportion tends to eliminate influence of the lost teeth on the final result.

Data from all patients, regardless of treatment procedure, were utilized in evaluating the overall effectiveness of pocket reduction and preservation of attachment level. Patient scores for the variables were calculated by averaging the scores assigned to the individual teeth. These patient scores were used for all statistical evaluations. The question to be answered by this analysis was how periodontal treatment affects the pocket depth and attachment level. Therefore, means were calculated for the change in pocket depth and attachment level at each of the yearly intervals up to 7 years. All subsequent scores of attachment level and pocket depth were related to the initial scores prior to treatment. Confidence intervals $(99 \%)$ were calculated primarily to test sig- 
TABLE I

Total $(M+D+B+L)$ Gain or Loss (-) of Attachment in

\begin{tabular}{cccccc} 
Years & Patients & Mean & S.D. & S.E. & " $t "$ \\
\hline 1 & 74 & 0.14 & 1.862 & 0.216 & 0.64 \\
2 & 78 & -0.28 & 1.779 & 0.201 & 1.37 \\
3 & 61 & -0.15 & 1.736 & 0.222 & 0.67 \\
4 & 38 & -1.20 & 2.296 & 0.372 & $3.23^{* *}$ \\
5 & 28 & -1.30 & 2.561 & 0.484 & $2.69 *$ \\
6 & 22 & -1.57 & 2.748 & 0.586 & $2.68 *$ \\
7 & 22 & -1.23 & 2.546 & 0.543 & $2.27^{*}$
\end{tabular}

TABLE II

Interproximal $(M+D)$ Gain or Loss $(-)$ of Attachment in $m$.

\begin{tabular}{cccccc} 
Years & Patients & Mean & S.D. & S.E. & "t" \\
\hline 1 & 74 & 0.37 & 1.115 & 0.129 & $2.86 * *$ \\
2 & 78 & 0.15 & 1.104 & 0.125 & 1.19 \\
3 & 61 & 0.30 & 0.992 & 0.127 & $2.33^{*}$ \\
4 & 38 & -0.17 & 1.308 & 0.212 & 0.82 \\
5 & 28 & -0.46 & 1.319 & 0.249 & 1.86 \\
6 & 22 & -0.46 & 1.304 & 0.278 & 1.67 \\
7 & 22 & -0.30 & 1.197 & 0.255 & 1.19 \\
& & & & & \\
& & & & Indicates $p<.05$ \\
& & & & Indicates $p<.01$
\end{tabular}

TABLE III

Buccal (B) or Loss (-) of At tachment in $=$.

\begin{tabular}{cccccc} 
Years & Patients & Mean & S.D. & S.E. & "t" \\
\hline 1 & 77 & -0.27 & 0.529 & 0.060 & $4.51 * *$ \\
2 & 80 & -0.37 & 0.543 & 0.061 & $6.05 * *$ \\
3 & 62 & -0.40 & 0.505 & 0.064 & $6.31 * *$ \\
4 & 39 & -0.74 & 0.589 & 0.094 & $7.79 * *$ \\
5 & 30 & -0.74 & 0.706 & 0.129 & $5.74 * *$ \\
6 & 23 & -0.90 & 0.755 & 0.157 & $5.71 * *$ \\
7 & 22 & -0.75 & 0.712 & 0.152 & $4.91 * *$
\end{tabular}

** Indicates $p<.01$ nificant changes from the initial zero score but could also be utilized as a conservative test of significant differences between means from year to year.

For testing differences in results from curettage and pocket elimination surgery, only the "split mouth" patients were utilized in analysis. The differences between the means were tested utilizing a Student "t" test. Confidence intervals $(99 \%)$ ) were also computed for these means. These C.I. are most useful in determining significant differences from zero while the power of the Student " $t$ " makes it a more appropriate test for distinguishing differences between two means.

The initial data for calculus, plaque, gingivitis, pocket depth and attachment levels for the teeth in the curettage and the teeth in the surgical pocket elimination groups were compared, and no statistically significant differences were found between the mean scores for the groups. It thus appears that the experimental sample was adequately balanced.

\section{Changes in attachment levels and pocket depths}

The sums of the mean gain or loss of attachment for the four surfaces of all treated teeth with time is reported in Table 1 .

It appears that one year following completion of the initial treatment, there is a slight gain of total periodontal attachment as measured clinically. This is followed by a slight loss during the second and third post-treatment year, and a statistically significant, but not very extensive loss between the third and fourth post-operative year. Following the fourth post-operative year, there is no further significant loss of attachment.

In order to evaluate the results of the treatment more specifically, the data for the combined mesial and distal measurements were analyzed as indicated in Table II. Here a significant gain is found for the first and third post-operative year, while the slight loss 4 to 7 years after the treatment is not significant.

The data from the buccal measurements (Table III), indicate a statistically significant loss of attachment for all of the post-operative measurements. The greatest loss was between the third and fourth year. However, the mean loss over 7 years was less than one millimeter.

The changes in lingual attachment levels (Table IV) were insignificant, except for the fourth post-operative year which showed a mean loss of $0.31 \mathrm{~mm}$.

The sums of the mean pocket reduction for all four surfaces of all treated teeth (Table V) indicate a statistically significant, but diminishing pocket reduction up to 6 years post-operatively. The gradual return of 
TABLE IV

Lingual (L) Gain or Loss (-) of Attachment in $\mathrm{mm}$.
TABLE VII

Buccal (B) Pocket Reduction or Increase $(-)$ in $m$.

\begin{tabular}{cccccc} 
Years & Patients & Mean & S.D. & S.E. & "t" \\
\hline 1 & 74 & 0.02 & 0.472 & 0.055 & 0.44 \\
2 & 78 & 0.05 & 0.520 & 0.059 & 0.85 \\
3 & 61 & -0.08 & 0.531 & 0.068 & 1.13 \\
4 & 38 & -0.31 & 0.647 & 0.105 & $2.96 *$ \\
5 & 28 & -0.12 & 0.944 & 0.178 & 0.66 \\
6 & 22 & -0.24 & 1.092 & 0.233 & 1.05 \\
7 & 22 & -0.23 & 1.022 & 0.218 & 1.08 \\
& & & & &
\end{tabular}

\begin{tabular}{cccccc} 
Years & Patients & Mean & S.D. & S.E. & "t" \\
\hline 1 & 77 & 0.63 & 0.451 & 0.051 & $12.21 * *$ \\
2 & 80 & 0.45 & 0.435 & 0.049 & $9.31 * *$ \\
3 & 62 & 0.28 & 0.451 & 0.057 & $4.81 * *$ \\
4 & 39 & 0.10 & 0.562 & 0.090 & 1.17 \\
5 & 30 & -0.05 & 0.532 & 0.097 & 0.51 \\
6 & 23 & -0.13 & 0.573 & 0.119 & 1.09 \\
7 & 22 & -0.14 & 0.619 & 0.132 & 1.03
\end{tabular}

TABLE V

Total $(M+D+B+L)$ Pocket Reduction in .

\begin{tabular}{cccccc} 
Years & Patients & Mean & S.D. & S.E. & " ${ }^{\prime \prime}$ \\
\hline 1 & 74 & 4.45 & 1.640 & 0.190 & $23.36 * *$ \\
2 & 78 & 3.44 & 1.749 & 0.198 & $18.02 * *$ \\
3 & 61 & 2.85 & 1.803 & 0.231 & $12.37 * *$ \\
4 & 38 & 2.33 & 2.145 & 0.348 & $6.70^{* *}$ \\
5 & 28 & 1.38 & 1.738 & 0.328 & $4.20^{* *}$ \\
6 & 22 & 0.98 & 1.970 & 0.420 & $2.34 *$ \\
7 & 22 & 0.67 & 1.805 & 0.385 & 1.74
\end{tabular}

-Indicates $p<.05$

* Indicates $p<.01$

TABLE VIII

Lingual (L) Pocket Reduction in m.

\begin{tabular}{cccccc} 
Years & Patients & Mean & S.D. & S.E. & "t" \\
\hline 1 & 77 & 1.01 & 0.565 & 0.064 & $15.72^{* *}$ \\
2 & 80 & 0.76 & 0.715 & 0.080 & $9.55 * *$ \\
3 & 62 & 0.66 & 0.582 & 0.074 & $8.96 *$ * \\
4 & 39 & 0.52 & 0.647 & 0.103 & $5.07^{* *}$ \\
5 & 30 & 0.41 & 0.524 & 0.096 & $4.32^{* *}$ \\
6 & 23 & 0.17 & 0.638 & 0.133 & 1.24 \\
7 & 22 & 0.06 & 0.576 & 0.123 & 0.48
\end{tabular}

TABLE VI

Interproximal $(M+D)$ Pocket Reduction in mm.

\begin{tabular}{cccccc} 
Years & Patients & Mean & S.D. & S.E. & "t" \\
\hline 1 & 77 & 2.81 & 1.354 & 0.154 & 18.23 ** \\
2 & 80 & 2.23 & 1.437 & 0.160 & $13.87 * *$ \\
3 & 62 & 1.92 & 1.557 & 0.198 & 9.71 ** \\
4 & 39 & 1.71 & 1.827 & 0.296 & $5.84 * *$ \\
5 & 30 & 1.03 & 1.444 & 0.263 & $3.89 * *$ \\
6 & 23 & 0.94 & 1.617 & 0.337 & $2.80 *$ \\
7 & 22 & 0.71 & 1.460 & 0.311 & $2.29 *$
\end{tabular}

TABLE IX

Total $(M+D+B+L)$ Gain or Loss $(-)$ of Attacheent

for Curettage and Surgery in $\boldsymbol{m}$.

\begin{tabular}{c|cccc|c|cccc} 
Years & \multicolumn{4}{c}{ Curettage } & \multicolumn{4}{c}{ Difference } & \multicolumn{3}{c}{ Surgery } \\
\hline & Patients & Mean & S.D. & S.E. & & Patients & Mean & S.D. & S.E. \\
\hline 1 & 48 & 0.90 & 1.892 & 0.273 & $1.40^{* *}$ & 58 & -0.50 & 1.907 & 0.250 \\
2 & 45 & 0.38 & 1.747 & 0.260 & $1.08 *$ & 57 & -0.70 & 2.159 & 0.286 \\
3 & 37 & 0.62 & 1.731 & 0.284 & $1.01 *$ & 47 & -0.39 & 1.792 & 0.261 \\
4 & 19 & -0.73 & 2.311 & 0.530 & 0.89 & 30 & -1.62 & 3.372 & 0.433 \\
5 & 12 & -2.06 & 2.168 & 0.626 & 0.38 & 13 & -2.44 & 2.276 & 0.641 \\
6 & 9 & -2.31 & 1.961 & 0.654 & 0.31 & 9 & -2.62 & 1.650 & 0.550
\end{tabular}


Figure 1

\section{Mean Change in Attachment with $99 \%$ C.I. for Curettoge and Surgery $(M+D+B+L)$}

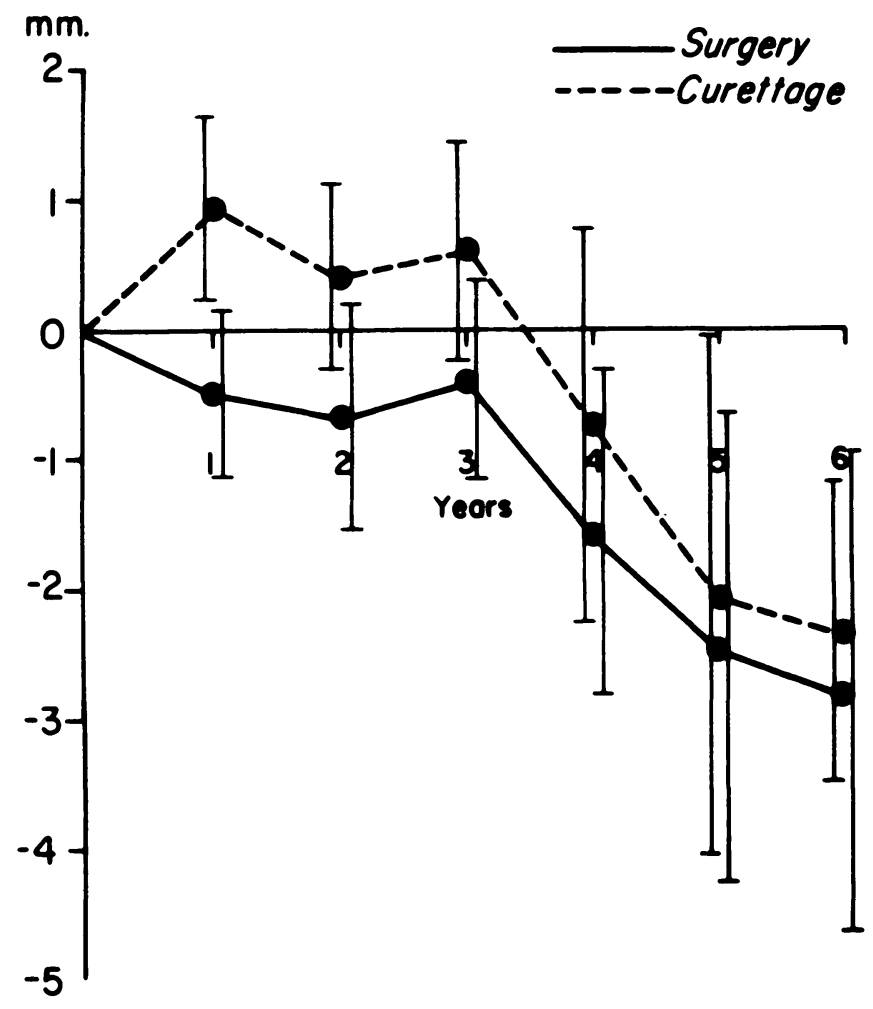

FIGURE 2

Mean Change in Attachment Level with 99\%C.I. for Curettage and Surgery $(M+D)$

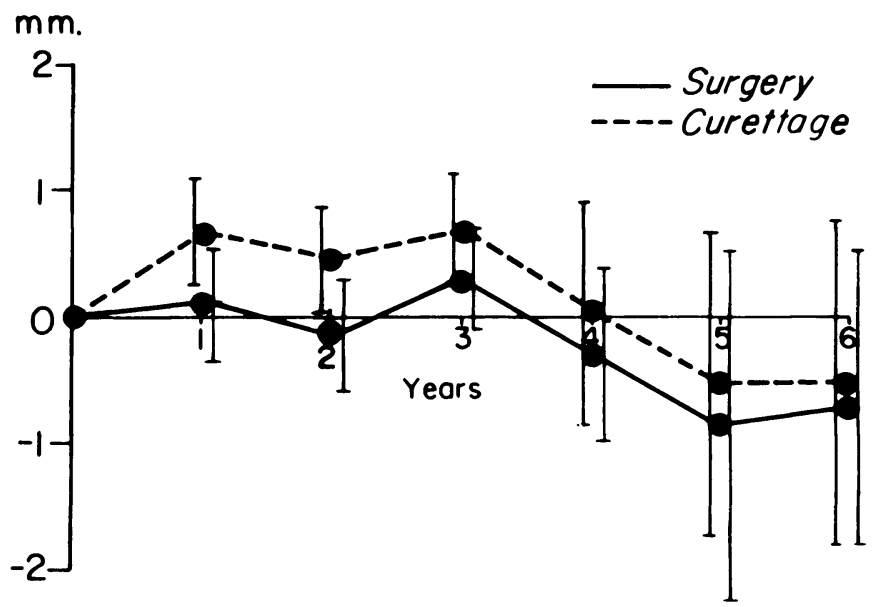

TABLE $X$

Interproximal $(M+D)$ Gein or Loss (-) Attachment for Curettage and Surgery in -

\begin{tabular}{c|cccc|c|cccc} 
Years & \multicolumn{4}{c}{ Curettage } & \multicolumn{4}{c}{ Difference } & \multicolumn{3}{c}{ Surgery } \\
\hline & Patients & Mean & S.D. & S.E. & & Patients & Mean & S.D. & S.E. \\
\hline 1 & 48 & 0.69 & 1.139 & 0.164 & $0.59^{*}$ & 58 & 0.10 & 1.173 & 0.154 \\
2 & 45 & 0.45 & 1.054 & 0.157 & $0.53^{*}$ & 57 & -0.08 & 1.233 & 0.163 \\
3 & 37 & 0.69 & 0.924 & 0.152 & 0.41 & 47 & 0.28 & 1.031 & 0.150 \\
4 & 19 & 0.01 & 1.311 & 0.301 & 0.29 & 30 & -0.28 & 1.342 & 0.245 \\
5 & 12 & -0.56 & 1.291 & 0.373 & 0.30 & 13 & -0.86 & 1.422 & 0.466 \\
6 & 9 & -0.52 & 1.164 & 0.388 & 0.21 & 0 & -0.73 & 1.037 & 0.346
\end{tabular}

pocket depth did not completely reach the preoperative level over 7 years of observation. The interproximal pocket reduction (Table VI) was significant for all of the seven post-operative years, although a gradual return of pocket depth occurred. The buccal pockets (Table VII) were reduced significantly for 3 years postoperatively, but gradually returned and became even slightly deeper than before treatment after 7 years. The mean pocket reduction on the lingual aspects of all treated teeth (Table VIII) was significant for 5 years post-operatively. However, by gradual return of pocket depth, the treated pockets reached very close to the pretreatment level after 7 years.

These composite results of two basically different approaches to periodontal therapy may also be evaluated and compared separately (Table IX). If measurements from all four surfaces of all treated teeth are added and compared, it appears that the attachment level is at a significantly more favorable level following curettage than following surgical pocket elimination up to 3 years following completion of the therapy. Over a longer period (4-6 years), there is some loss of attachment also following curettage but to a significantly lesser degree than following surgery. A comparison of results following the two experimental procedures is shown graphically in Figure 1. There is a significant loss of attachment 4-6 years following both curettage and surgical pocket elimination, although slightly less following curettage than following surgery. It should be understood that the average loss of attachment at any given surface of the teeth is less than one millimeter over 7 years.

If the combined loss or gain of mesial and distal attachment is considered (Table $\mathrm{X}$ ), the results are more favorable than when all surfaces were pooled together and the results from curettage are consistently better than for surgical pocket elimination (Figure 2), although the differences are small but statistically significant (p. $<0.05)$ for the first 2 post-operative years. 
TABDI $x$

Duccal Loes (-) of Attacheent for Curettage and Surgery in -

\begin{tabular}{|c|c|c|c|c|c|c|c|c|c|}
\hline \multirow{2}{*}{ Yeare } & \multicolumn{4}{|c|}{ Curettoge } & \multirow[t]{2}{*}{1 ff ferenc } & \multicolumn{4}{|c|}{ Sursery } \\
\hline & Patiente & Mean & S.D. & S.E. & & Pattente & Meen & S.D. & s.r. \\
\hline 1 & 50 & -0.10 & 0.544 & 0.077 & $0.30 * \star$ & 60 & -0.40 & 0.557 & 0.072 \\
\hline 2 & 46 & -0.28 & 0.564 & 0.083 & 0.17 & 58 & -0.45 & 0.632 & 0.003 \\
\hline 3 & 30 & -0.25 & 0.577 & 0.094 & $0.27 *$ & 48 & -0.52 & 0.462 & 0.067 \\
\hline 4 & 20 & -0.66 & 0.665 & 0.149 & 0.14 & 32 & -0.80 & 0.638 & 0.113 \\
\hline 5 & 14 & -0.73 & 0.636 & 0.169 & 0.28 & 15 & -1.01 & 0.684 & 0.177 \\
\hline 6 & 10 & -0.96 & 0.582 & 0.184 & 0.27 & 10 & -1.23 & 0.648 & 0.205 \\
\hline
\end{tabular}

* Indicates $p<.05$

TABLE XII

Lingual (L) Galn or Loes $(-)$ of Attachant

\begin{tabular}{|c|c|c|c|c|c|c|c|c|c|}
\hline aere & & Curet & & & Ifferenc & & Surs & & \\
\hline & Patcents & Mean & S.D. & S.E. & & Patlente & Keen & S.D. & 3.2. \\
\hline 1 & 48 & 0.33 & 0.571 & 0.082 & $0.54 * *$ & 58 & -0.21 & 0.531 & 0.070 \\
\hline 2 & 45 & 0.16 & 0.553 & 0.082 & $0.37^{* *}$ & 57 & -0.21 & 0.629 & 0.003 \\
\hline 3 & 37 & 0.15 & 0.686 & 0.113 & $0.32 \star$ & 47 & -0.17 & 0.636 & 0.093 \\
\hline 4 & 19 & -0.05 & 0.810 & 0.186 & $0.46 *$ & 30 & -0.51 & 0.670 & 0.122 \\
\hline 3 & 12 & -0.69 & 0.633 & 0.183 & 0.11 & 13 & -0.38 & 0.627 & 0.174 \\
\hline 6 & 9 & -0.80 & 0.771 & 0.257 & 0.00 & 9 & -0.80 & 0.581 & 0.193 \\
\hline
\end{tabular}

TABLE XIII

Total $(M+D+B+L)$ Pocket Reduction or Increase $(-)$ for Curettage and Surgery in

\begin{tabular}{l|cccc|c|cccc} 
Years & \multicolumn{4}{c}{ Curettage } & \multicolumn{3}{c}{ Difference } \\
\hline & Patients & Mean & S.D. & S.E. & & Patients & Mean & S.D. & S.E. \\
\hline 1 & 50 & 3.85 & 2.020 & 0.285 & $1.22^{* *}$ & 60 & 5.07 & 1.664 & 0.215 \\
2 & 46 & 3.02 & 1.918 & 0.283 & $0.89^{*}$ & 58 & 3.91 & 1.694 & 0.222 \\
3 & 38 & 2.72 & 1.997 & 0.324 & 0.65 & 48 & 3.37 & 1.735 & 0.250 \\
4 & 20 & 1.82 & 2.124 & 0.475 & 0.41 & 32 & 2.23 & 2.520 & 0.445 \\
3 & 14 & 0.25 & 1.679 & 0.449 & $1.64 *$ & 15 & 1.89 & 1.887 & 0.487 \\
6 & 10 & -0.10 & 1.685 & 0.533 & 1.41 & 10 & 1.31 & 1.808 & 0.572
\end{tabular}

* Indicates $p<.05$
Indicates $p<.01$

TABLE XIV

Interproximal $(M+D)$ Pocket Reduction for Curettage and Surgory in -

\begin{tabular}{l|cccc|c|cccc} 
Years & \multicolumn{4}{c}{ Curettage } & \multicolumn{4}{c}{ Difference } & \multicolumn{3}{c}{ Surgery } \\
\hline & Patients & Mean & S.D. & S.E. & & Patients & Mean & S.D. & S.E. \\
\hline 1 & 50 & 2.53 & 1.649 & 0.233 & $0.63^{*}$ & 60 & 3.16 & 1.336 & 0.172 \\
2 & 46 & 2.02 & 1.557 & 0.230 & 0.48 & 58 & 2.50 & 1.388 & 0.182 \\
3 & 38 & 1.95 & 1.723 & 0.280 & 0.26 & 48 & 2.21 & 1.425 & 0.206 \\
4 & 20 & 1.44 & 1.711 & 0.383 & 0.20 & 32 & 1.64 & 2.135 & 0.377 \\
5 & 14 & 0.70 & 1.409 & 0.377 & 0.74 & 15 & 1.44 & 1.439 & 0.372 \\
6 & 10 & 0.71 & 1.285 & 0.406 & 0.66 & 10 & 1.37 & 1.438 & 0.455
\end{tabular}

- Indicates $p<.05$
FIGURE 3

Meon Change in Attachment Level with 99\% C. I. for Curettage and Surgery (Buccal)

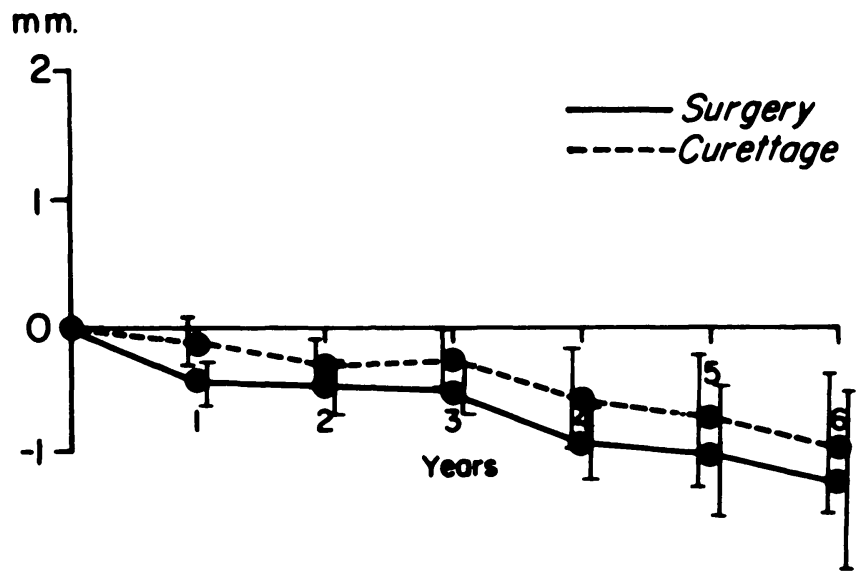

FIGURE 4

Mean Change in Attochment Level with 99\% C. I. for Curettage and Surgery (Lingual)

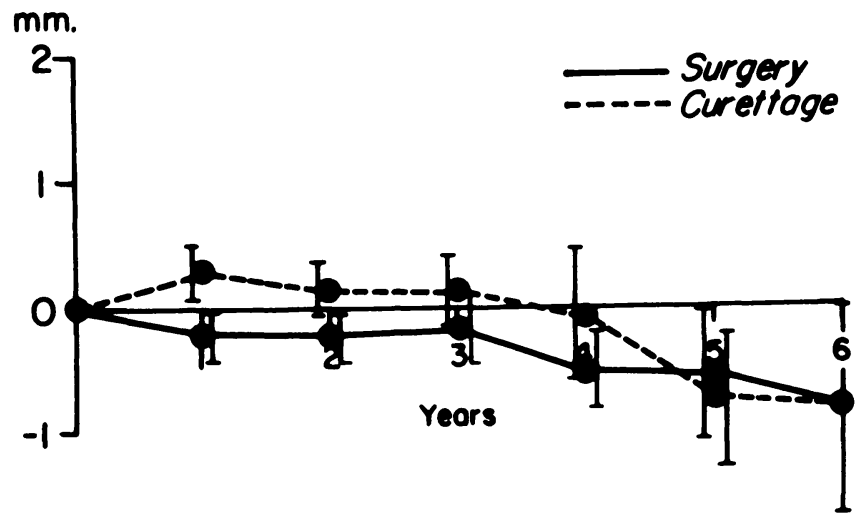

The average loss for each mesial or distal surface is only $1 / 4 \mathrm{~mm}$. over 6 years following curettage and slightly more following surgical pocket elimination.

On the buccal aspect of the teeth there was a slowly progressing but statistically significant loss of attachment both following curettage and surgical pocket elimination with no remarkable differences between the two procedures (Table XI and Figure 3 ). The total average loss was about one millimeter over 6 years for the buccal surfaces, which is considerably more than for the interproximal surfaces.

The end results, over 5-6 years, of the lingual measurements of attachment levels are similar to the buccal results (Table XII and Figure 4). However, for the first four post-operative years, the results following curet- 
FIGURE 5

Mean Pocket Reduction with $99 \%$ C.I. for Cureffoge and Surgery $(M+D+B+L)$

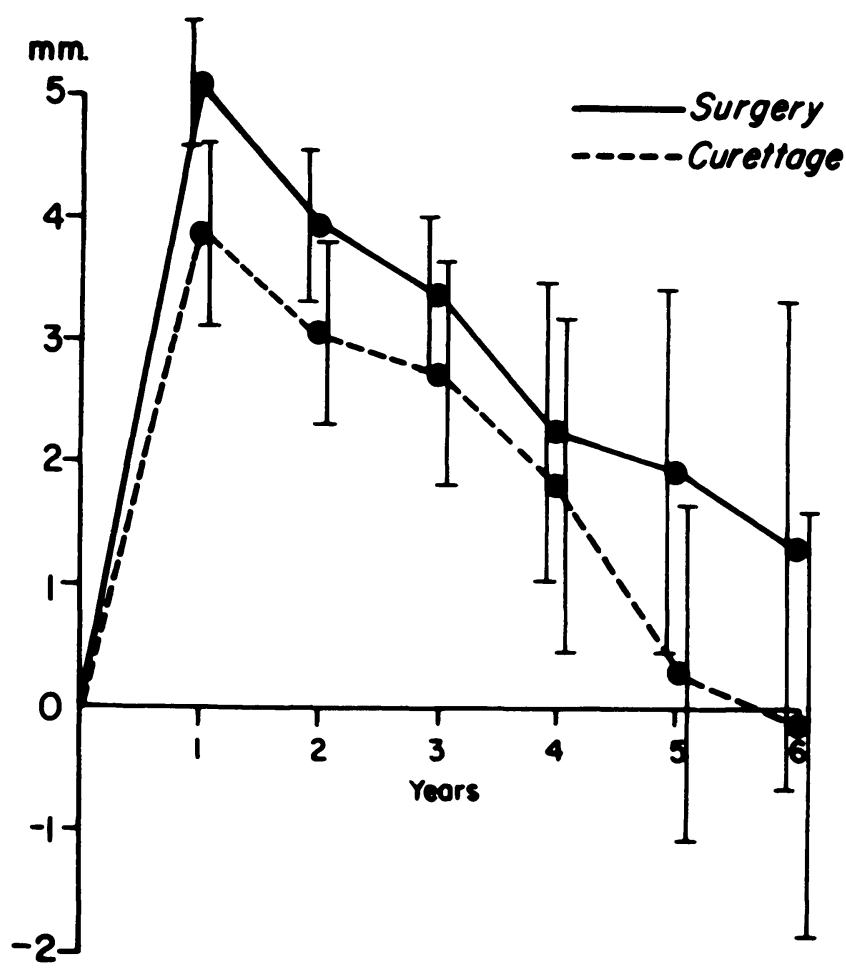

FIGURE 6

Meon Pocket Reduction with $99 \%$ C.I. for Curetfoge and Surgery (M+D)

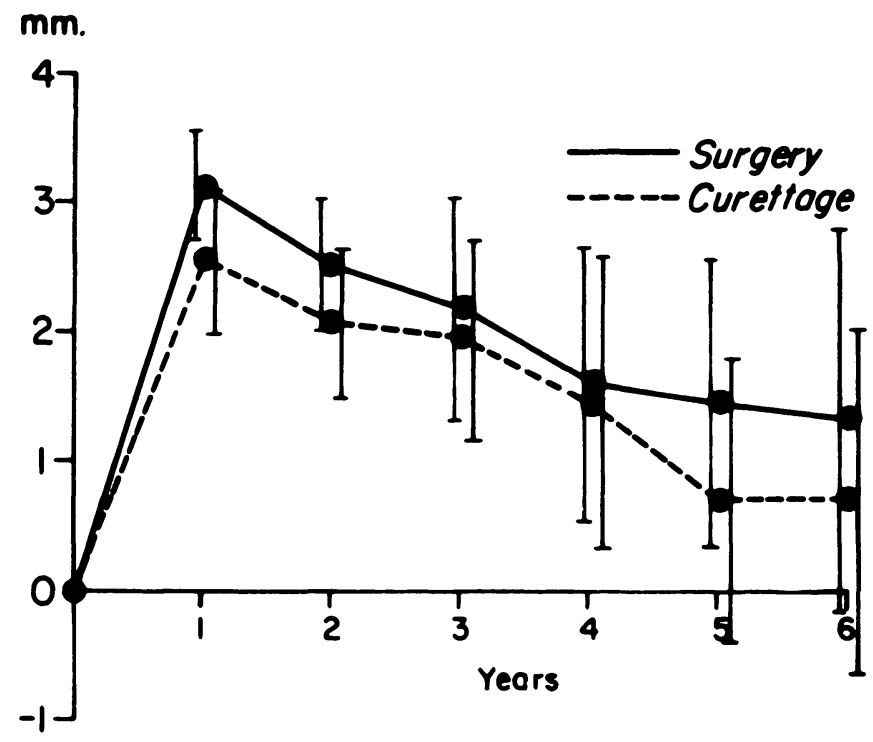

TABLE XY

Duccal (B) Pocket Roduction or Increase $(-)$ for Curettage and Surgory in

\begin{tabular}{l|cccc|c|cccc} 
Years & \multicolumn{4}{c}{ Curettage } & \multicolumn{4}{c}{ Difference } & \multicolumn{3}{c}{ Surgery } \\
\hline & Patients & Mean & S.D. & S.E. & & Pationts & Mean & S.D. & S.E. \\
\hline 1 & 50 & 0.48 & 0.584 & 0.083 & $0.23^{*}$ & 60 & 0.71 & 0.561 & 0.072 \\
2 & 46 & 0.37 & 0.538 & 0.079 & 0.12 & 58 & 0.49 & 0.493 & 0.065 \\
3 & 38 & 0.25 & 0.523 & 0.085 & 0.07 & 48 & 0.32 & 0.427 & 0.062 \\
4 & 20 & -0.07 & 0.573 & 0.128 & 0.13 & 32 & 0.06 & 0.642 & 0.113 \\
5 & 14 & -0.31 & 0.568 & 0.152 & 0.21 & 15 & -0.10 & 0.622 & 0.161 \\
6 & 10 & -0.42 & 0.480 & 0.152 & 0.12 & 10 & -0.30 & 0.625 & 0.196 \\
& & & & & & & & & \\
\end{tabular}

TABLE XVI

Lingunal (L) Pocket Reduction or Increase $(-)$ for Curettage and Surgery in -

\begin{tabular}{c|cccc|c|cccc} 
Yoars & \multicolumn{4}{c}{ Curettege } & \multicolumn{3}{c}{ Difference } & \multicolumn{3}{c}{ Surgery } \\
\hline & Patients & Mean & S.D. & S.E. & & Patients & Mean & S.D. & S.L. \\
\hline 1 & 50 & 0.84 & 0.691 & 0.097 & $0.36^{* *}$ & 60 & 1.20 & 0.671 & 0.006 \\
2 & 46 & 0.64 & 0.696 & 0.102 & $0.28^{*}$ & 58 & 0.92 & 0.624 & 0.062 \\
3 & 38 & 0.51 & 0.667 & 0.108 & $0.33^{*}$ & 48 & 0.84 & 0.715 & 0.103 \\
4 & 20 & 0.44 & 0.803 & 0.179 & 0.09 & 32 & 0.53 & 0.830 & 0.147 \\
5 & 14 & -0.13 & 0.427 & 0.114 & $0.67 *$ & 15 & 0.54 & 0.790 & 0.204 \\
6 & 10 & -0.39 & 0.698 & 0.228 & 0.65 & 10 & 0.26 & 0.690 & 0.218
\end{tabular}

$\therefore$ Indicates $p<.05$

tage are significantly better than following surgical elimination statistically, although these differences may be of dubious clinical significance. The total loss over 6 years approaches one millimeter for both procedures.

Pocket reduction and return of pocket depth were also compared following curettage and surgical pocket elimination. When the combined measurements from all four surfaces are considered (Table XIII, and Figure 5), it appears that although there was a significantly greater pocket reduction for the first two years post-operatively, the pocket depth returned at approximately the same rate following both procedures (see Figure 5).

The interproximal pocket reduction and return in pocket depth were fairly similar following the two procedures (Table XIV and Figure 6) although the reduction in depth was greater for the surgical elimination. The pocket depth did not return to the pretreatment level for either of the procedures over the 6 years of observation.

For the buccal aspects, the pocket reduction and rate of return also were similar following the two procedures (Table XV, and Figure 7). Five to six years post-operatively the average pocket depth was greater than prior to the treatment for both modalities of treatment.

The pocket reduction on the lingual aspect of the teeth was consistently greater following surgical pocket elimination than following curettage (Table XVI, and 
FIGURE 7

Mean Pocket Reduction with $99 \%$ C.I. for Curetfoge and Surgery (Buccol)
FigURE 8

Meon Pocket Reduction with $99 \%$ C.I. for Curetfoge and Surgery (Lingual)

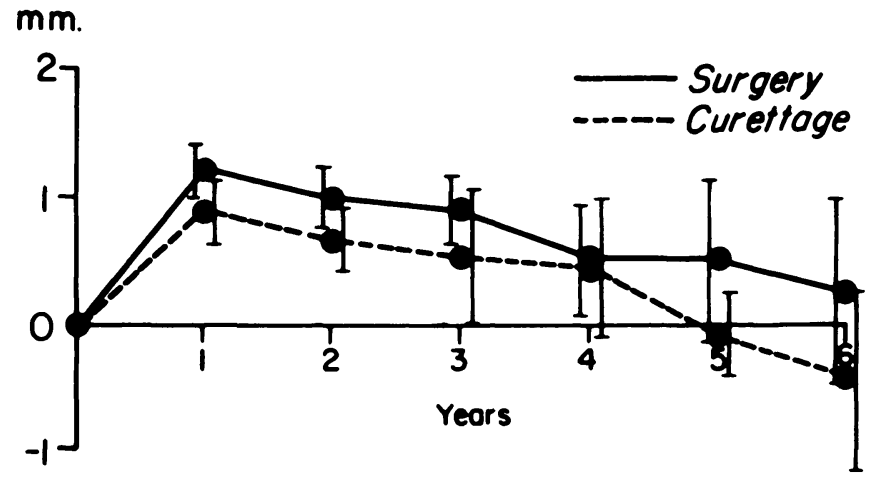

FIGURE 10

Mean Pocket Reduction and Change in Attochment Level with $99 \%$ C.I. (M+D) All Patients

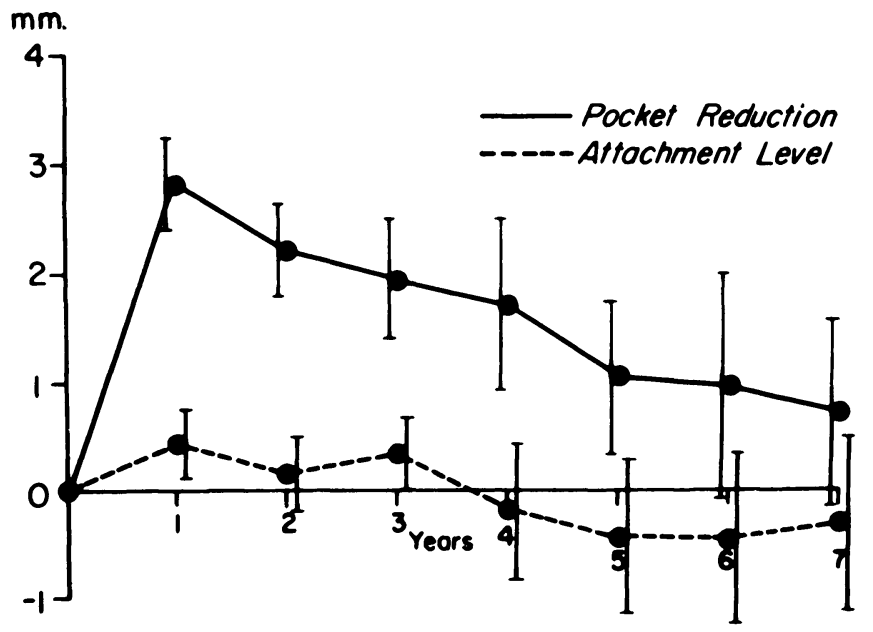

FIGURE 11

Mean Pocket Reduction and Change in Attochment Level with $99 \%$ C.I. (Buccol)

All Potients

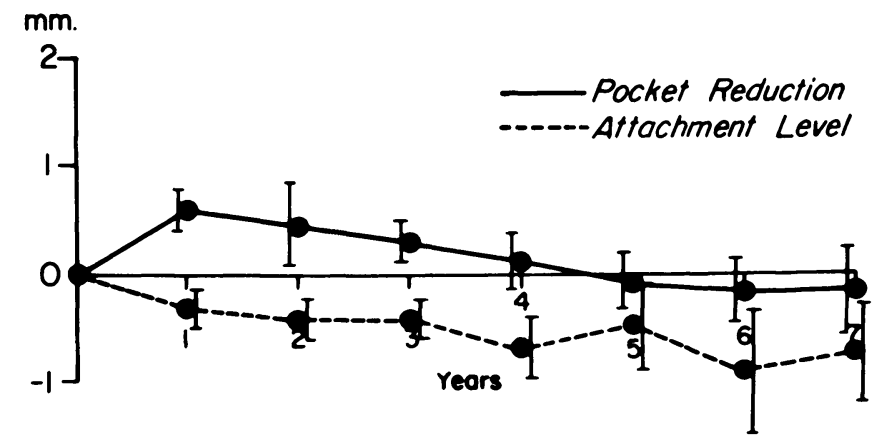


FIGURE 12

\section{Mean Pocket Reduction and Change in Attochment Level with $99 \%$ C.I. (Lingual) \\ All Patients}

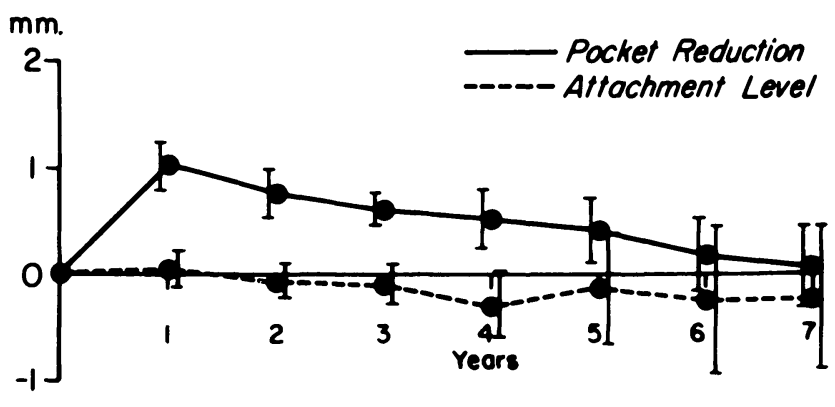

Figure 8). In four of the six years of observation these differences were statistically significant (Table XVI), but the curves appear to follow a very similar path (see Figure 8).

\section{Attachment levels related to variations in pocket depth}

In order to evaluate the significance of reduction of pocket depth to the maintenance of the attachment level, data on pocket reduction and attachment levels for all of the treated teeth were compared (Figure 9). In general, it may be stated that pockets were reduced in depth and the attachment level improved one year after treatment; then to be followed by a gradual return of pocket depth and, to a lesser degree, a gradual loss of attachment. This trend is evident even if the results are separated for the interproximal (Figure 10), the buccal (Figure 11), and the lingual (Figure 12) measurements. However, a more detailed study of the variations in measurements shows several exceptions to this general trend, for example in the interproximal areas (Figure 10) where there are several deviations between the course of the curves for pocket depth and attachment levels.

Since it is well known that pocket depth increases and attachment is lost with time, it is not possible to draw any conclusion concerning cause and effect relationship by simply comparing this type of measurement.

A more meaningful approach seems to be a comparison of the results following curettage and following surgical pocket elimination. The surgical pocket elimination therapy reduced the pocket depth significantly more than curettage (Table XIII). However, the response of the attachment level was more favorable after curettage than after surgical pocket elimination (Table IX). Although these differences were not statistically significant for the long term observation, the trend was con- sistent throughout the study. It thus appears that the degree of elimination or reduction of clinically probeable pockets is not related directly to the maintenance of periodontal attachments.

\section{Significance}

\section{Attachment levels}

The progression of the periodontal disease was stopped fairly well for a period of 3 years post-operatively regardless of modality of treatment (Table I). The main exception was the buccal aspects of the teeth (Table III) which showed a significant loss. These short term results are even more encouraging if the interproximal areas are considered separately (Table II) since these are usually the areas with the deepest periodontal pockets. ${ }^{6}$ It also appears that over 3 years curettage gave significantly better results than surgical pocket elimination (Table IX) with regards to gain or loss of attachment.

However, during long term observation (4-7 years) there was a statistically significant, although for each surface only about $0.3 \mathrm{~mm}$. loss, of attachment. The progressive loss from the fourth to the seventh year was not significant. The long term losses were not significantly different following curettage and surgical pocket elimination (Table IX). This significant loss of attachment from the third to the fourth post-operative year has been a consistent observation during the present study. It is apparently very important that longitudinal studies on the results of periodontal therapy are extended beyond 3 years of observation. It may also be indicated to retreat areas which show tendency for breakdown 3-4 years after the initial treatment. In the present study the patients were given only routine maintenance care by a dental hygienist and the progressing lesions were not reoperated.

The rate of progress of untreated periodontal disease for various age groups is not well known. Data from recent studies ${ }^{4,5}$ suggest that for younger individuals not receiving specific prophylactic care, the average loss of attachment may approximate $0.1 \mathrm{~mm}$. for each tooth surface per year. Even if such data were available for general populations they would not be applicable to the selected group of patients in this study with much more than average advanced periodontal disease for their age.

Since for ethical and practical reasons, pockets could not be left untreated, the absolute value of the periodontal treatment cannot be assessed from this study. However, the short term gain, and the long term slight loss of periodontal attachment for these patients with advanced periodontal disease establishes a more favorable prognosis for treatment of advanced periodontal lesions than 
generally conceived, especially when the minimal loss of teeth from periodontal disease (less than 1 per cent) during the entire experimental periods is considered.

The role of the initial occlusal adjustment, as well as the 3 month prophylaxis in the total result of the treatment cannot be assessed. The main conclusions regarding clinically measurable attachment levels stated on the basis of the experimental design of the present study are: 1. A short term (1-3 years) gain of periodontal attachment following subgingival curettage may be anticipated. 2. There is a tendency towards a significant loss of attachment to occur between 3 and 5 years following both curettage and surgical pocket elimination. 3. Even for long term (4-7 years) observation, the average loss of attachment is minimal from a clinical standpoint (about $0.3 \mathrm{~mm}$. over 7 years). 4 . The long term effect (4-7 years) on attachment levels is not significantly different following curettage and surgical pocket elimination. 5. The loss of teeth (with advanced periodontal disease) is minimal following treatment (less than 1 percent in the present study).

\section{Pocket depth}

A significant reduction in total pocket depth occurred following both experimental procedures (Table $\mathrm{V}$ and Fig. 6). However, a gradual return of pocket depth reduced the significance level until the differences were not significant after 7 years. The most significant pocket reduction was interproximally (Table VI), and least buccally (Table VII).

The pocket reduction was greater following surgical elimination than following curettage. The differences were significant for total pocket depth (Table XIII) for 2 years post-operatively and close to significant levels for the other post-operative years. The most significant difference was for the lingual pockets (Table XVI) and least for the buccal (Table XV).

Conclusions regarding pocket reduction: 1 . There is a significant reduction of pocket depth both following surgical elimination and following curettage (Figure 5), and a trend towards gradual return of pocket depth (Figure 9). 2. The reduction of pocket depth is greater and sustained better following surgical elimination than following curettage (Figure 5).

3. Significance of pocket reduction for maintenance of attachment levels.

The interplay between reduction with subsequent partial return of pocket depth with the variations in attachment levels is difficult to approach from a statistical basis. However, the fact that one method of treatment (surgical elimination) reduced the pocket depth most and the other type of treatment (curettage) gave the most favorable results regarding attachment levels should indicate that there is not a direct dependent relationship between variations in pocket depth and attachment levels.

It appears that pocket reduction and elimination may not be as essential a feature of successful periodontal therapy as generally assumed, providing the patient receives adequate periodontal maintenance care. This is clinically a very important observation since surgical pocket elimination will result in more root exposure than curettage and thus create more esthetic, hygiene, and functional problems.

4. Discussion of the post-operative gain or loss of attachment.

The loss of the attachment which was gained following curettage, especially in the interproximal areas (Figure 2), may be related to a long, thin, post-operative epithelial attachment which reopened as a result of irritation as suggested by Gottlieb ${ }^{7}$ in 1927 , and by others later. ${ }^{8}$

A logical sequella of the present study would be to retreat areas which show clinical signs of marked inflammation and opening of pockets to see if loss of attachment could be prevented or attachment regained.

\section{SUMMARY}

Two common approaches to periodontal therapy, curettage and surgical pocket elimination followed by periodic prophylaxes, have been applied to 104 patients over a period of from one to ten years.

The range of age at the initiation of the treatment was 13 to 64 years, with a mean age of 39.7 years (S.D. 13.54). There were 50 females and 54 males. The average depth of the interproximal pockets at the distobuccal line angle of all teeth was $4.04 \mathrm{~mm}$. (S.D. 1.766), and at the mesio-buccal line angle $3.93 \mathrm{~mm}$. (S.D. 1.717). The average loss of attachment at the same location was respectively $3.53 \mathrm{~mm}$. (S.D. 2.140), and $3.14 \mathrm{~mm}$. (S.D. 2.011). A total of 2,604 teeth have been treated in these 104 patients, averaging approximately 25 teeth per patient.

The variations in attachment levels related to the cementum-enamel junction, and pocket depth have been assessed in millimeters once a year.

Evaluation of the results indicates that:

1. Over a short term observation (1-3 years), curettage resulted in a slight gain of attachment, while there was a slight loss following surgical pocket elimination. 
2. A significant loss of attachment occurred between 3 and 5 years following completion of the treatment for both experimental groups.

3. The long-term (4-7 years) loss of attachment was not significantly different for the two groups.

4. Pocket reduction was greater and sustained better following surgical elimination than following curettage.

5. Degrees of pocket reduction did not relate directly to variations in attachment levels.

\section{BIBLIOGRAPHY}

1. Ramfjord, S. P., et al: Subgingival curettage versus surgical elimination of periodontal pockets. J. Periodont., 39:167-175, 1968.

2. Gladvin, L. and Löe, H.: Errors in the clinical as- sessment of periodontal destruction. J. Periodont. Res., 2:180, 1967.

3. Smith, L. W., et al: A study of intra-examiner variation in scoring oral hygiene status, gingival inflammation and epithelial attachment level. J. Periodont., 41:671, 1970.

4. Suomi, J. D., et al: The effect of controlled oral hygiene procedures on the progression of periodontal disease in adults: Results after third and final year. J. Periodont., 42:152, 1971.

5. Lightner, L. M., et al: Preventive periodontic treatment procedures; Results over 46 months. J. Periodont., 42:555, 1971.

6. Lövdal, A., Arnö, A., and Waerhaug, J.: Incidence of clinical manifestations of periodontal disease in light of oral hygiene and calculus formation. J.A.D.A., 56:21, 1958.

7. Gottlieb, B.: Tissue changes in pyorrhoea. J.A.D.A., 14:2178, 1927.

8. Ramfjord, S. P.: Experimental periodontal reattachment in rhesus monkeys. J. Periodont., 22:67, 1951.

\section{Abstracts}

Human Gingival Collagenase: Purification, Molecular Weight, and INHibitor Studies

Fullmer, H. M., Taylor, R. E., and Guthrie, R. W.

J. Dent. Res., 51:349, 1972

Human gingival collagenase derived from culture fluids was subjected to ammonium sulfate precipitation and column chromatography. Collagenase assays were carried out using labeled collagen obtained from embryonic chick calvaria which was added to unlabeled rat skin collagen. Inhibitor studies were carried out on varying concentrations of human serum. A molecular weight for collagenase of approximately 4,000 was obtained from both the Sephadex G-75 column and Sepharose 6 B columns. Total inhibition of human gingival collagenase activity was observed with inhibitors which completely removed calcium, sulfhydryl compounds, human serum, and glutaraldehyde. Institute of Dental Research, School of Dentistry, University of Alabama in Birmingham, Birmingham, Alabama.

\section{An Alteration In Human Diabetic Arterioles}

Keene, J. J., Jr.

J. Dent. Res., 51:569, 1972

Palatal biopsy specimens obtained from 17 nondiabetic and 12 diabetic patients were excised from clinically normal regions and stained with aldehyde fuchsin (AF), acid orcein (AO) and subjected to hyaluronidase digestion as well as routine hematoxylin and eosin staining to evaluate vascular morphology. In diabetics AO-positive and AF-positive deposition was found in the region circumscribing the media as well as the internal elastic membrane of the tunica intima. This deposition was believed to be elastic tissue. Eleven of the 12 diabetics, and five nondiabetic specimens contained arterioles with pronounced
AF- and AO-positive material appearing in the adventitia which were distinctly separate from the internal elastic membrane. Arteriolar widths in these specimens were generally thickened. Not all arterioles in any particular diabetic specimen showed the indicated vascular changes. Department of Oral Pathology and Periodontology, Loyola University, School of Dentistry, Maywood, Illinois 60153.

Penicillin-The Duration of Its Activity In Blood Clots Juniper, R. P.

Brit. J. Oral Surg., 9:222, March, 1972

Blood clots were removed from tooth sockets of 20 patients who had had multiple extractions under general anesthesia. Ten patients (Group I) were given a single preoperative intramuscular dose of $600 \mathrm{mg}$. of penicillin $\mathrm{G}$ at the time of premedication prior to extraction. The Group II patients had a single dose of the same preparation administered approximately 15 hours after the extractions; and this was followed by two subsequent intramuscular four ml. increments of Procaine Penicillin, Fortified, B.P. at seven hour intervals. Blood clots were removed at six, twelve, and 24 hours after the initial injection in both groups. Samples of serum and saliva were also collected with each specimen of clot for determination of their penicillin concentrations. The cup-plate assay method was employed using Sarcina Lutea (ATCC 9341) as the test organism. The results showed that in both groups therapeutic concentrations of penicillin in blood clots formed in tooth sockets were reached, but that without reinforcement, the concentration of penicillin in the clot had dropped to zero within 12 hours. From the results in Group II it was shown that penicillin diffused rapidly into the preformed clots but that frequent reinforcement was necessary to maintain it at a therapeutic concentration. Queen Victoria Hospital, East Grinstead, Sussex, England. 